\title{
SCIDiC
}

International Journal of Dentistry and Oral Science (IJDOS)

ISSN: 2377-8075

\section{The Effectiveness Of Amine Fluoride Mouthwash In Preventing White Spot Lesions During Fixed Orthodontic Therapy: A Randomized Control Trial}

Research Article

Ravikiran K.R ${ }^{1}$, Sharanya Sabrish, MDS $^{2}$, Silju Mathew, MDS 3 , Prashantha G.S, MDS ${ }^{4}$, Roshan Sagarkar ${ }^{5}$

${ }^{1}$ Faculty of Dental Sciences, M S Ramaiah University of Applied Sciences, MSRIT Post, M S R Nagar, Bengaluru, Karnataka, India.

${ }^{2}$ Reader, Department of Orthodontics, Faculty of Dental Sciences, M S Ramaiah University of Applied Sciences, MSRIT Post, M S R Nagar, Bengaluru, Karnataka, India.

${ }^{3}$ Professor \& HOD, Department of Orthodontics, Faculty of Dental Sciences, M S Ramaiah University of Applied Sciences, MSRIT Post, M S R Nagar, Bengaluru, Karnataka, India.

${ }^{4}$ Professor, Department of Orthodontics, Faculty of Dental Sciences, M S Ramaiah University of Applied Sciences, MSRIT Post, M S R Nagar, Bengaluru, Karnataka, India.

${ }^{5}$ Reader, Department of Orthodontics, Faculty of Dental Sciences, M S Ramaiah University of Applied Sciences, MSRIT Post, M S R Nagar, Bengaluru, Karnataka, India.

\section{Abstract}

Background and Aims: One of the main reasons that patients seek orthodontic treatment is esthetics. Unfortunately, treatment with fixed orthodontic appliances can at times increase the chances of developing white spot lesions on the surfaces of teeth, thus creating a new esthetic issue for the patient.The prevalence of white spot lesions (WSL) reported after treatment varies from $2 \%$ to $97 \%$, and these lesions can occur in spite of increased attention to hygiene. Hence the aim of this study was to assess the effect of Amine fluoride mouthwash in preventing white spot lesions during fixed orthodontic therapy.

Materials \& Methods: Study participants were randomly divided in to 2 groups. (Group A -experimental 25 patients and Group B -control 25 patients). The pre-intervention and post-intervention intra oral digital photographs were analyzed by using Image $J$ software to assess the percentage of the facial surface affected on the teeth (10 maxillary teeth) which had white spot lesions.

Results: The mean value of WSLs showed significant difference between the experimental and control groups. Mean values of WSL in the experimental group decreased from 2 to 1.54 with a mean difference of 0.46 after 6 months follow-up which indicated that amine fluoride mouthwash was effective in preventing WSLs during fixed orthodontic treatment.

Conclusion: WSLs scores were significantly reduced in patients who received the mouthwash when compared to patients who followed Standard oral hygiene regimen with fluoridated toothpaste only.

Keywords: White Spot Lesions; Amine Fluoride; Fluoride Mouthwash; Fixed Orthodontic Treatment.

\section{Introduction}

One of the main reasons that patients seek orthodontic treatment is esthetics.[1] Unfortunately, treatment with fixed orthodontic appliances can at times increase the chances of developing white spot lesions on the surfaces of teeth, thus creating a new esthetic issue for the patient.[1]T he prevalence of white spot lesionsassociated with orthodontic treatment has been reported to be variable from $2 \%$ to $97 \%$, and they tend to develop in spite of increased attention to oral hygiene.[2], [3] This has been attributed to many reasonssuch as changes in plaque microorganisms, increase in plaque retention areas due to the appliance, difficulty in maintaining hygiene, and reduction of natural cleansing mechanisms.[2] Development of white spot lesions requires a fermentable carbohydrate, bacteria, enamelsurface and time. Fixed appliances tend to trap food debris and plaque control also becomes difficult. This can lead to increased acid production by the plaque bacteria andsubsequent demineralization. [4] Also, the plaque can

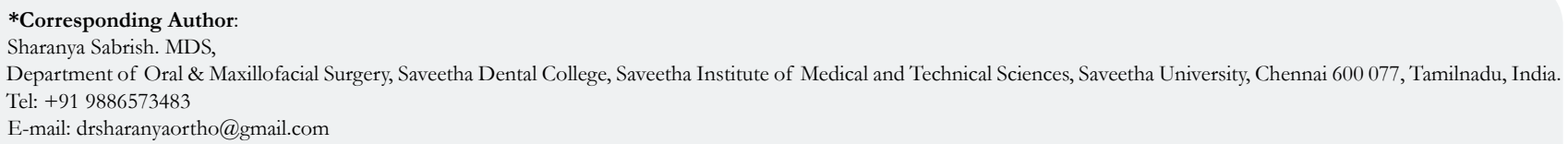

Citation: Ravikiran K.R, Sharanya Sabrish, MDS, Silju Mathew, MDS, Prashantha G.S, MDS, Roshan Sagarkar. The Effectiveness Of Amine Fluoride Mouthwash In Preventing White Spot Lesions During Fixed Orthodontic Therapy: A Randomized Control Trial. Int I Dentistry Oral Sci. 2021;8(7):3486-3490.

doi: http://dx.doi.org/10.19070/2377-8075-21000712

Copyright: Sharanya Sabrish. MDS ${ }^{\circ} 221$. This is an open-access article distributed under the terms of the Creative Commons Attribution License, which permits unrestricted use, distribution and reproduction in any medium, provided the original author and source are credited. 
act as a barrier to prevent the acid diffusion away from the enamel surface and mineral diffusion towards the enamel, thusfavoring demineralization. Fixed orthodontic appliances are associated with increased numbers of Streptococcus mutans and Lactobacilli, microorganisms which have been linked to increased caries incidence. [5] Once formed, white spot lesions can compromise esthetics and are difficult or even impossible to reverse. Saliva can help to remineralize the lesion to some degree, but this process is slow and rarely results in complete resolution of the lesion. Fluoride application has been shown to increase the rate of remineralization of such lesions. [6]

High doses of fluoride have been recommended to aid in preventing decalcification and preventing WSLs from progressing to carious lesions during and after orthodontic treatment.[7] Some authors have recommended not to use high concentrations of fluoride because they believe that this will cause remineralization only in the superficial part of the WSLs and this will prevent calcium and phosphate from penetrating into the deeper layers of the enamel, inhibiting the remineralization and limiting the esthetic improvement. [8] In order to use the available fluoride preparations and procedures optimally, the clinician must be familiar with the mechanisms ofaction of fluoride.[9] Systematic and narrative reviews have presented various strategies to prevent WSL development and there is evidence that topical fluoride (varnishes, gels, toothpaste) or fluoride containing bonding material can reduce the severity of WSL formation adjacent to fixed appliances.[10] A Study conducted by Arnold and Leonard et al concluded that significant reduction in enamel WSLs could be achieved during orthodontic therapy with $0.2 \%$ sodium fluoride rinse. [10] A Study conducted by Mikael and Svante et al proved that the daily use of high fluoride tooth paste can significantly reduce the prevalence and incidence of lesions adjacent to fixed orthodontic appliances. [10] Othersreports suggested that topical fluoride varnish application during treatment with fixed orthodontic appliances can reduce the development of lesions adjacent to brackets.[12] Similarly, Philip E Benson and Parkin et al showed that use of fluoride containing bonding materials can reduce the occurrence and severity of white spot lesions.[13] There was no detailed published information found on the effect of Amine fluoride mouthwash (Amflor, Group Pharma, India) in reducing the incidence of white spot lesions after orthodontic bonding. Amflor contains amine fluoride, which is an organic fluoride as the chief ingredient. It offers an active remineralization process to strengthen enamel. The ph of amine fluoride is slightly acidic and due to this fluoride ions can rapidly combinewith calcium in dental enamel to form calcium fluoride and this improves the remineralization process.

Hence the aim of this study was to assess the effectiveness of amine fluoride mouthwash in preventing thedevelopment of white spot lesions during fixed orthodontic therapy.

\section{Materials and Methods}

This study was conducted at the Department of Orthodontics, Faculty of Dental Sciences, Ramaiah University of Applied Sciences, Bangalore, with the approval of the Institutional Ethical Committee. This was a randomized (1:1), double blinded, active controlled, parallel group trial. The inclusion criteria were: $\mathrm{Pa}-$ tientsin the age bracket of 12-28 years, low caries risk individu- als [14] (low risk refers to no previous white spot lesions, good oral hygiene, low dietary sugar exposure, etc), patients who were scheduled for treatment with fixed orthodontic appliances for an expected duration of at least 1 year, patients who agreed not to use any additional oral hygiene products for the whole study duration other than the ones received in the study and the patients who agreed to sign the written informed consent. The exclusion criteria were: known hypersensitivity/allergy to study products and /or materials used, if they had undergone professional administration of highly concentrated fluoride within 30 days prior to enrollment, any alteration in enamel for example: hypoplasia, fluorosis, multiple restoration on facial surface, chronic use of any medications causing dry mouth or xerostomia, subjects with special needs such as syndromes, cleft lip and palate deformities, etc. Sample size estimation was carried out and it was deduced that a sample size of 50 subjects would yield $80 \%$ power in order to detect significant differences, with the effect size of 0.8 and significance level set at 0.05 . Randomization and allocation was carried out. The study design is presented in figure 1 . Study participants were randomly divided in to 2 groups. (Group A -experimental group with 25 patients and Group B -control group with 25 patients) using the lottery method.Control Group (A): Patients were instructed to follow the regular oral hygiene procedure: brushing twice daily with fluoridated toothpaste.Study Group (B): Patients were instructed to follow both the regular oral hygiene procedure (brushing twice daily along with fluoridated toothpaste) and additionally daily use of amine fluoride mouthwash (amine fluoride of $480 \mathrm{ppm})$.

The study was conducted from the commencement of bonding procedure and a follow-up over a period of 6-months was done. Routine pre-bonding cleaning procedures were followed and M.B.T 0.022 inch slot brackets (TP Ortho Nu-Edge) were bonded and excess composite material was removed with an explorer. Concealment was carried out. The examiner distributed unlabeled containers containing amine fluoride mouthwash to the experimental group and a placebo to the control group. Patients were instructed to follow an organized oral hygiene protocol. Verbal instructions as well as printed home care directions were provided. Subjects were instructed to usethe mouth rinse once daily before bedtime by swishing $15 \mathrm{ml}$ of undiluted mouthwash for a duration of 25-30 seconds vigorously and then spitting it out. Mouthwash quantity was measured by using dosimeter provided and the compliance was monitored by printed calendar provided on the bottle back. Participants were instructed to mark the day they did not follow mouthwash regime.

Digital intraoral photographs were taken with the patient's head tilted up approximately 5 to 10 degree in order to limit reflection from the flash to the maxillary incisors. Several photographs were taken to allow selection of the optimal image. Finally, 3 images were taken for each case - 1 frontal view, 1 right lateral view and 1 left lateral view. Photographs were taken before bondingand 6 months after bonding brackets. The pre-intervention and postintervention intra oral digital photographs were analyzed by using Image J software version $1.50 \mathrm{i}$ as shown in figure 2 . The examiner was trained and calibrated in the department of orthodontics for the usage of Image J software 1.50i (Java) for estimating white spot lesions on 10 participants who were not included in the final study sample.The facial surfaces of 10 maxillary teeth (premolar to premolar) were evaluated for lesions. The percentage of the facial surface affected was calculated on the teeth which had lesions. 
The teeth without WSLs were recorded having zero percentage.

The data collected was entered in a Microsoft Excel file and statistical analyses were performed using the Statistical Package for Social Science (SPSS version 18.5) software. The normality of the data was assessed using Shapiro Wilk test. Mann Whitney U test was applied to assess the difference between the two groups. Proportions were compared using the chi-square test of significance.

\section{Results}

The mean age of the participants between the study groups was compared and is shown in table 1. Student's t test was applied to compare the mean age between the study groups. No difference was found. Table 2 compares the gender distribution in both experimental and control group which also showed no difference. The tests of normality were carried out and it was found that the data was not normally distributed. So all comparisons between the groups were carried out using a non-parametric test.

Table 3 shows the comparison of Mean value of WSLs between Study groups during pre and post-intervention using MannWhitney Test. The $\mathrm{p}$ value was 0.068 before intervention in both control and experimental group which indicates the WSLS scores were not significantly different before intervention. The mean value of WSLs in the experimental group after 6 months follow-up was 1.54 and $p$ value was 0.006 , which is statistically significant. Table 4 shows the comparison of Mean value of Pre and Postintervention WSLs values within the Study group. Wilcoxon Sign Rank Test was applied to compare of Mean value of Pre and Post-interventions of WSL scores. Mean value of WSLs showed significant difference in both experimental and control groups. Within the control group a difference of -0.55 was noted, the negative value indicates that the WSL scores increased significantly in the group who were instructed to follow standard oral hygiene regimen with fluoridated toothpaste. However, the mean values of WSL within the experimental group decreased from 2 to 1.54 after 6 months follow-up which indicates that the amine fluoride mouthwash was effective in preventing the WSLs during fixed orthodontic treatment.

Table 5 demonstrates the student t test which was applied to assess the change from Mean Pre-intervention WSLs values to Postintervention WSLs values between the groups. Change in mean values of WSLs prior to orthodontic treatment and during 6 months follow-up showed a significant mean difference of -0.01 between control and experimental groups. The mean difference value obtained at the end of study was -0.01 which is $<$ than 0.001 which is considered to be statistically significant.

Figure 1. Randomized controlled trial study design.

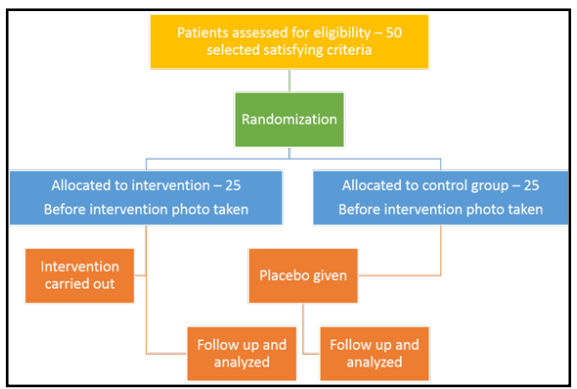

Figure 2. The area of WSL measured using the tools of the software, full area versus affected area to calculate the percentage.

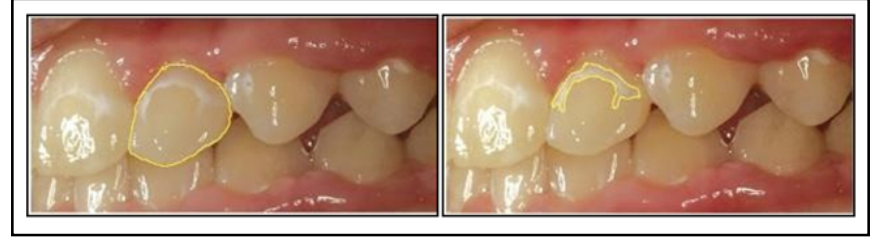

Table 1. The comparison of the mean age between groups.

\begin{tabular}{|c|c|c|c|c|c|c|c|}
\hline & N & Mean & SD & Min. & Max. & 't' value* & 'p' value \\
\hline Control & 25 & 20.6 & 4.795 & 14 & 28 & \multirow{2}{*}{1.194} & \multirow{2}{*}{0.28} \\
\hline Experiment & 25 & 19.2 & 4.518 & 14 & 28 & & \\
\hline
\end{tabular}

Table 2. Comparison of gender between groups.

\begin{tabular}{|c|c|c|c|c|c|}
\hline & \multicolumn{2}{|c|}{ Gender } & \multirow{2}{*}{ Total } & \multirow{2}{*}{ c2 value* } & P value \\
\cline { 2 - 4 } & Male & Female & & & \\
\hline \multirow{2}{*}{ Control } & 13 & 12 & 25 & & \\
\cline { 2 - 4 } & $52.00 \%$ & $48.00 \%$ & $100.00 \%$ & & \multirow{2}{*}{0} \\
\hline \multirow{2}{*}{$\begin{array}{c}\text { Experi- } \\
\text { ment }\end{array}$} & 13 & 12 & 25 & 1 \\
\cline { 2 - 4 } Total & $52.00 \%$ & $48.00 \%$ & $100.00 \%$ & & \\
\cline { 2 - 4 } & 26 & 24 & 50 & & \\
\hline
\end{tabular}


Table 3. Comparison of Mean value of WSLs between Study groups during Pre and Post-intervention using Mann- Whitney Test.

\begin{tabular}{|c|c|c|c|c|c|c|c|c|c|}
\hline & & $\mathbf{N}$ & Mean & SD & Median & Min. & Max. & value* & 'p' value \\
\hline \multirow{3}{*}{ Pre Treatment } & Control & 25 & 1.91 & 1.939 & 1.22 & 0.41 & 10.15 & \multirow{2}{*}{210.5} & \multirow{2}{*}{0.068} \\
\cline { 2 - 9 } & Experiment & 25 & 2 & 1.022 & 1.55 & 0.9 & 4.19 & & \\
\hline \multirow{2}{*}{ Post-intervention } & Control & 25 & 2.46 & 1.868 & 1.78 & 0.89 & 9.92 & \multirow{2}{*}{170.5} & \multirow{2}{*}{0.006} \\
\cline { 2 - 8 } & Experiment & 25 & 1.54 & 0.835 & 1.1 & 0.81 & 3.4 & & \\
\hline
\end{tabular}

Table 4. Comparison of Mean value of WSLs, Pre and Post-intervention values within the Study groups. Wilcoxon Sign Rank Test was applied to compare of Mean value of Pre and Post-interventions of WSL scores within the Study groups.

\begin{tabular}{|c|c|c|c|c|c|c|c|c|c|}
\hline Group & Treatment & $\mathbf{N}$ & Mean & SD & Median & Mean Diff & SE Diff & Z value* & P value \\
\hline \multirow{2}{*}{\begin{tabular}{c} 
Control \\
\cline { 2 - 10 }
\end{tabular}} & Pre & 25 & 1.91 & 1.939 & 1.22 & -0.55 & 0.087 & -4.292 & $<0.001$ \\
\cline { 2 - 10 }$n$ & Post & 25 & 2.46 & 1.868 & 1.78 & & & & \\
\hline \multirow{2}{*}{$\begin{array}{c}\text { Experi- } \\
\text { ment }\end{array}$} & Pre & 25 & 2 & 1.022 & 1.55 & 0.46 & 0.075 & -4.029 & $<0.001$ \\
\cline { 2 - 7 } & Post & 25 & 1.54 & 0.835 & 1.1 & & & & \\
\hline
\end{tabular}

Table 5. The student $t$ test which was applied to assess the change from Mean Pre-intervention WSLs values to Post-inter. vention WSLs values between the groups.

\begin{tabular}{|c|c|c|c|c|c|c|c|}
\hline Group & N & Mean & SD & Mean Diff & SE Diff & t value* & P value \\
\hline Control & 25 & -0.55 & 0.437 & \multirow{2}{*}{-1.01} & 0.115 & -8.763 & $<0.001$ \\
\hline Experiment & 25 & 0.46 & 0.373 & & & & \\
\hline
\end{tabular}

\section{Discussion}

White spot lesions are a serious problem in orthodontics. Brackets and other orthodontic appliances make oral hygiene more difficult. The clearance of plaque adjacent to the attachments by saliva and the cheeks are reduced.[15] Carious lesions tend to develop in such sites where microbes are free to form plaque that is not disrupted by mechanical abrasion during routine tooth brushing. [16] There are many methods and instruments for assessing and analyzing the presence and severity of enamel WSLs. Commonly used methods are the non-destructive method, Opacity index, micro radiographic and SEM examinations, CCD camera with diffuse laser light, clinical photography, direct clinical examination, quantitative laser fluorescence and Diagnodent. In this study, digital intraoral photographic method was selected for assessing enamel WSLs, because this was considered feasible and reliable. However, QLF, Diagnodent and CCD camera can also be used for assessing the lesions, but they are expensiveand cumbersome to use. Chapman J et al compared the results of photographs and direct clinical examination and observed that there was $99.2 \%$ correlation between these two methods.[16] According to them there are differences in the refractive index of air (1.00), water (1.33) and enamels crystals (1.66). Hence we can deduce that those lesions that require air-drying to become visible (opaque) have lost less amount of minerals than a lesion which is visible without being air-dried. This has been confirmed by histological studies which detected lesser porosities in lesions visible only after being air-dried in comparison to lesions which are visible without being air-dried. [11]

In this study, the incidence and severity of white spot lesions on the upper anterior teeth (right premolar to left premolar) were evaluated by assessing pre and post-intervention intraoral photographs of 50 patients. Using Image J 1.50i software the area of WSLs were calculated on individual tooth surfaces. Mean value of WSLs within the control group showed a difference of -0.55 , the negative value suggests that the WSL scores increased significantly in the group who followed only the standard oral hygiene regimen with fluoridated toothpaste. Whereas, the WSL scores decreased during 6 months follow-up from 2 to 1.54 which indicates that amine fluoride mouthwash was effective in preventing white spot lesions during fixed orthodontic treatment. The mean value of WSLs prior to orthodontic treatment and during 6 months follow-up was also assessed and it showed significant mean difference of -0.01 between control and experimental groups, which is considered statistically significant. The results showed that the prevalence of WSLs in the experimental group were lesser when compared to control group (Standard oral hygiene regimen with fluoridated toothpaste).

Patient compliance with this mouthwash is a major factor to be considered in its success, and hence it was reinforced by constant motivation and regular clinical follow- ups of the patients. From the results obtained from the study we can deduce that, low dose of Amine fluoride mouthwash was effective in prevention of white spot lesions during fixed orthodontic treatment over a period of 6 months follow-up. WSLs scores were significantly reduced in patients who received amine fluoride mouthwash when compared to patients who followed standard oral hygiene regimen with fluoridated toothpaste.

\section{Conclusion}

Low dose of Amine fluoride mouthwash is an effective method for prevention of WSLs, during fixed orthodontic treatment over 
a 6 months follow-up.On macroscopic examination of maxillary incisors and premolars, the WSLs scores were significantly reduced in patients who received Amflor mouthwash when compared to patients who followed Standard oral hygiene regimen with only fluoridated toothpaste.

\section{References}

[1]. Huang GJ, Roloff-Chiang B, Mills BE, Shalchi S, Spiekerman C, Korpak AM, et al. Effectiveness of MI Paste Plus and PreviDent fluoride varnish for treatment of white spot lesions: a randomized controlled trial. Am J Orthod Dentofacial Orthop. 2013 Jan;143(1):31-41. Pubmed PMID: 23273358.

[2]. Torlakovic L, Klepac-Ceraj V, Ogaard B, Cotton SL, Paster BJ, Olsen I. Microbial community succession on developing lesions on human enamel. J Oral Microbiol. 2012;4. Pubmed PMID: 22432048.

[3]. Gorelick L, Geiger AM, Gwinnett AJ. Incidence of white spot formation after bonding and banding. Am J Orthod. 1982 Feb;81(2):93-8. Pubmed PMID: 6758594.

[4]. Srivastava K, Tikku T, Khanna R, Sachan K. Risk factors and management of white spot lesions in orthodontics. J Orthod Sci. 2013 Apr;2(2):43-9. Pubmed PMID: 24987641.

[5]. Øgaard B. White spot lesions during orthodontic treatment: mechanisms and fluoride preventive aspects. InSeminars in orthodontics 2008 Sep 1 (Vol. 14, No. 3, pp. 183-193). WB Saunders.

[6]. Zachrisson BU, Zachrisson S. Caries incidence and orthodontic treatment with fixed appliances. Scand J Dent Res. 1971;79(3):183-92. Pubmed PMID: 5285724.

[7]. O'Reilly MM, Featherstone JD. Demineralization and remineralization around orthodontic appliances: an in vivo study. Am J Orthod Dentofacial Orthop. 1987 Jul;92(1):33-40. Pubmed PMID: 3300270.
[8]. García-Godoy F, Hicks MJ. Maintaining the integrity of the enamel surface: the role of dental biofilm, saliva and preventive agents in enamel demineralization and remineralization. J Am Dent Assoc. 2008 May;139 Suppl:25S34S. Pubmed PMID: 18460677.

[9]. Øgaard B, Larsson E, Henriksson T, Birkhed D, Bishara SE. Effects of combined application of antimicrobial and fluoride varnishes in orthodontic patients. Am J Orthod Dentofacial Orthop. 2001 Jul;120(1):28-35. Pubmed PMID: 11455374

[10]. Sonesson M, Twetman S, Bondemark L. Effectiveness of high-fluoride toothpaste on enamel demineralization during orthodontic treatment-a multicenter randomized controlled trial. Eur J Orthod. 2014 Dec;36(6):678-82. Pubmed PMID: 24375756.

[11]. Geiger AM, Gorelick L, Gwinnett AJ, Benson BJ. Reducing white spot lesions in orthodontic populations with fluoride rinsing. Am J Orthod Dentofacial Orthop. 1992 May;101(5):403-7. Pubmed PMID: 1590288.

[12]. Stecksén-Blicks C, Renfors G, Oscarson ND, Bergstrand F, Twetman S. Caries-preventive effectiveness of a fluoride varnish: a randomized controlled trial in adolescents with fixed orthodontic appliances. Caries Res. 2007;41(6):455-9. Pubmed PMID: 17827963.

[13]. Benson PE, Parkin N, Millett DT, Dyer FE, Vine S, Shah A. Fluorides for the prevention of white spots on teeth during fixed brace treatment. Cochrane Database Syst Rev. 2004;(3):CD003809. Pubmed PMID: 15266503.

[14]. Heymann GC, Grauer D. A contemporary review of white spot lesions in orthodontics. J Esthet Restor Dent. 2013 Apr;25(2):85-95. Pubmed PMID: 23617380.

[15]. Abou Neel EA, Aljabo A, Strange A, Ibrahim S, Coathup M, Young AM, et al. Demineralization-remineralization dynamics in teeth and bone. Int J Nanomedicine. 2016 Sep 19;11:4743-4763. Pubmed PMID: 27695330.

[16]. Chapman JA, Roberts WE, Eckert GJ, Kula KS, González-Cabezas C. Risk factors for incidence and severity of white spot lesions during treatment with fixed orthodontic appliances. Am J Orthod Dentofacial Orthop. 2010 Aug;138(2):188-94. Pubmed PMID: 20691360 